\title{
3-D Printed Anti-Reflection Structures for the Terahertz Region
}

\author{
Jarosław Bomba $^{1} \cdot$ Jarosław Suszek $^{1} \cdot$ Michał Makowski $^{1}$ (D) \\ Artur Sobczyk $^{1} \cdot$ Maciej Sypek $^{1}$
}

Received: 9 May 2017 / Accepted: 28 August 2017 /

Published online: 6 September 2017

(C) The Author(s) 2017. This article is an open access publication

\begin{abstract}
Terahertz radiation has a growing number of applications in material characterization, where spectral fingerprinting and diffractive effects are the carriers of information. On the other hand, electromagnetic waves in the range of millimeters exhibit strong unwanted specular reflections, resulting in uncontrolled interferences. This problem is especially disturbing in the goniometric time-domain spectroscopy (TDS) configuration, where angular distribution of the field modified by the sample is altered by unwanted reflections. For this reason, low-cost anti-reflection layers are desired. Here, we present a simple way of designing and manufacturing one-sided and two-sided anti-reflection polyamide layers for the $\mathrm{THz}$ range. The structures were fabricated using 3-D printers based on selective laser sintering. We demonstrate experimentally in the goniometric time-domain spectroscopy the significant reduction of wavelength-dependent oscillations in Fabry-Perot configuration in the range between 0.1 and $0.3 \mathrm{THz}$. We also examine the influence of the anti-reflection layers on the distribution of THz energy in reflected, transmitted, and diffracted fields.
\end{abstract}

Keywords/OCIS codes (310.1210) Anti-reflection · (040.2235) Far infrared or terahertz • (300.6495) Spectroscopy, terahertz $\cdot(110.6795)$ Terahertz imaging

\section{Motivation}

Terahertz radiation has been the subject of growing attention because of its unique properties: non-ionizing nature, good transmission through many non-metallic media, and the possibilities of spectral fingerprinting of various materials, including hazardous ones. THz beams are used in numerous areas, including medicine, telecommunication, and security systems. In most of

Michał Makowski

michal.makowski@if.pw.edu.pl

1 Faculty of Physics, Warsaw University of Technology, 75 Koszykowa, PL00662 Warszawa, Poland 
the application scenarios, the information is carried by spectral distribution, therefore systems providing a linear spectral response from 0.1 to $3 \mathrm{THz}$ are highly desired.

Beams with wavelengths in the range of millimeters are specularly reflected from object surfaces. Moreover, multiple reflections occur in slabs of materials and the phasing of such multiple wavefronts gives raise to Fabry-Perot (F-P) effects. For these reasons, spectral measurements of flat samples are usually compromised by the periodic attenuation function, resulting from the F-P phenomenon.

Stray reflections from flat slabs of materials can be partly suppressed by adding antireflection (AR) coatings [1,2]. The traditional multi-layer approach in this case is problematic due to the small choice of feasible materials with appropriate refractive indices [3-5]. Brückner et al. have examined the use of TOPAS, PTFE, and HDPE materials structured with single-point diamond turning [1] and ultraprecision turning process [6] for reduced reflections. Recently, all dielectric microstructured materials have been successfully used as THz absorbers [7]. Gatesman and Hübers have validated the use of parylene films as AR layers; nevertheless, their use below $1 \mathrm{THz}$ is troublesome due to problems with proper thickness [8, 9]. Recently, Neeraj et al. have proposed the use of metallic copper structures as AR for $\mathrm{THz}$ for the price of non-negligible amplitude attenuation [10]. In this paper, we present the use of cost-effective, dielectric 3-D printed anti-reflection coatings. The design is straightforward and the precision of additive manufacturing ensures the proper functionality for the frequency range of $0.1-0.3 \mathrm{THz}$.

\section{The Test Configuration}

The proposed 3-D printed AR structures were tested in the straightforward configuration of a plastic slab of material illuminated at an angle of $45^{\circ}$ with a collimated $\mathrm{THz}$ beam, which usually exhibits strong F-P oscillations [11].

The test configuration is shown in Fig. 1. The plastic slab with a refractive index of $n$ and width of $d$ is illuminated at an angle $\alpha$ to the normal with a single ray $A_{1}$. As a result of multiple reflections from the plastic-air interface, numerous $T_{n}$ rays are transmitted through the slab and simultaneously $R_{n}$ rays are reflected from the slab.

The resultant rays are mutually coherent, therefore phase relations between subsequent rays must be established in order to assess the final reflectance and transmittance of the slab as a function of the wavelength. The following formula gives the phase delay between rays $T_{1}$ and $T_{2}[12]:$

$$
\Delta=4 \pi \frac{n}{\lambda} d \cos \beta
$$

while the transmission of such etalon plate can be described by:

$$
T_{e}=\frac{1}{1+F \sin ^{2}\left(\frac{\Delta}{2}\right)},
$$

where, $F=\frac{4 R}{(1-R)^{2}}$ is the finesse and $R$ is the reflectance of both surfaces. Figure 2 shows the theoretical transmission of the slab for different finesse values.

Obviously, the maximal transmission occurs when the optical path difference (OPD) between subsequent transmitted rays is equal to the multitude of the wavelength. 
Fig. 1 Ray tracing of $\mathrm{THz}$ beam illuminating the test plastic slab, resulting in Fabry-Perot interference

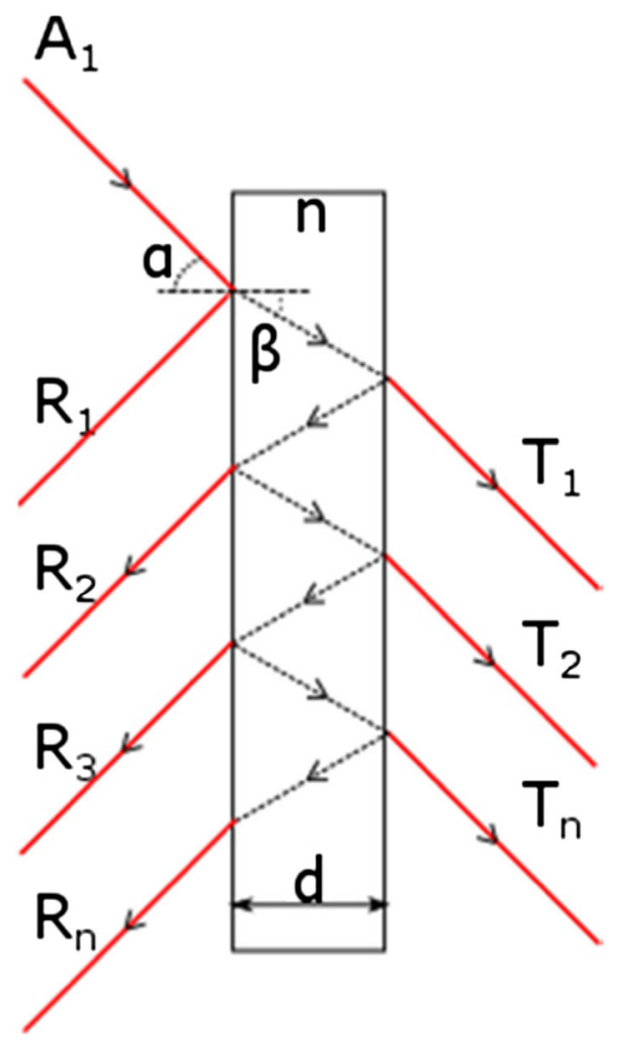

\section{Design and Fabrication of THz Anti-Reflection Coatings}

The working principle of the proposed AR coating is the formation of a gradient of the refractive index at the interface between the air and the plastic slab. The precision of currently available additive manufacturing allows 3 -D printing of diffractive $[13,14]$ and sub-

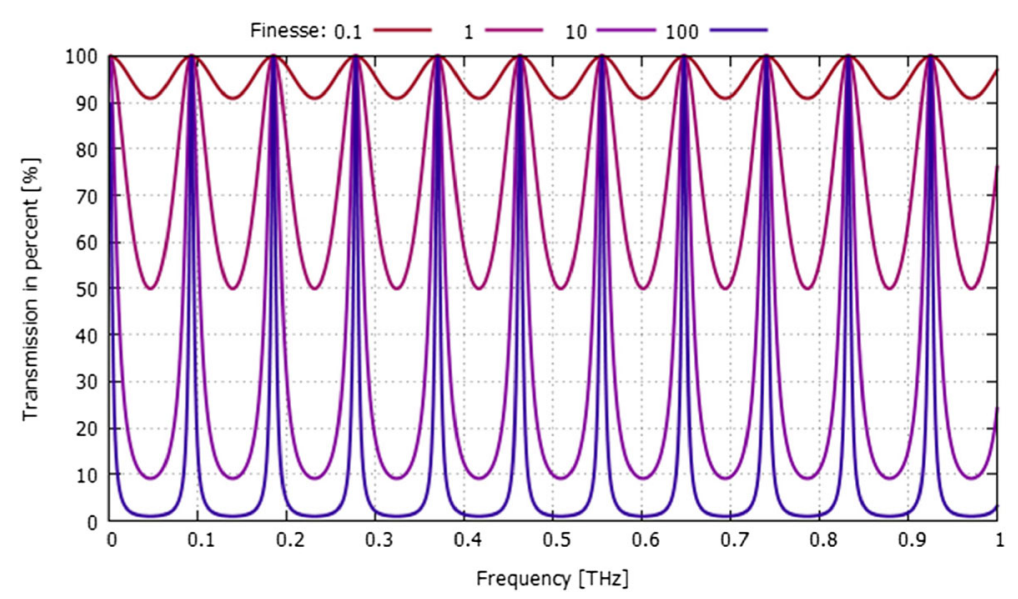

Fig. 2 Theoretical transmission of a slab for four different finesse values 

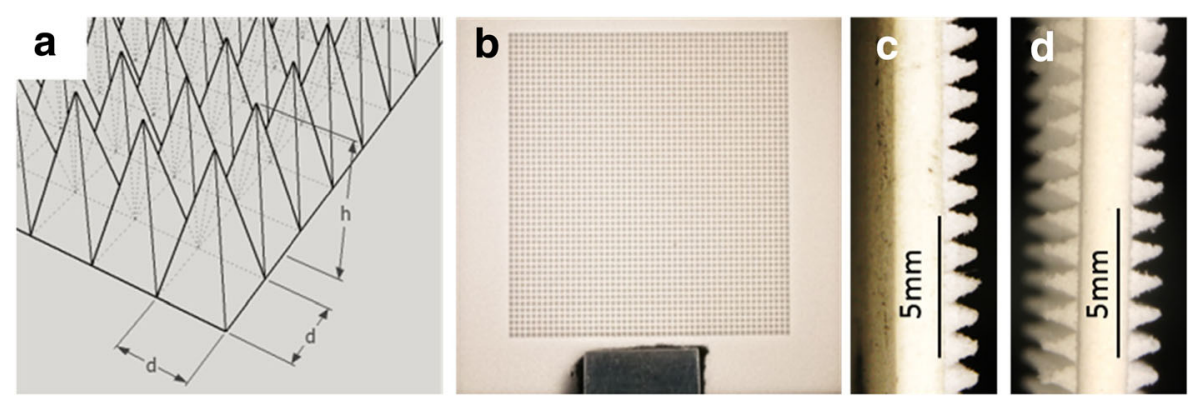

Fig. 3 Anti-reflection coatings. a Design. b Photo. c Close-up of a one-sided AR layer. d Close-up of a twosided AR layer
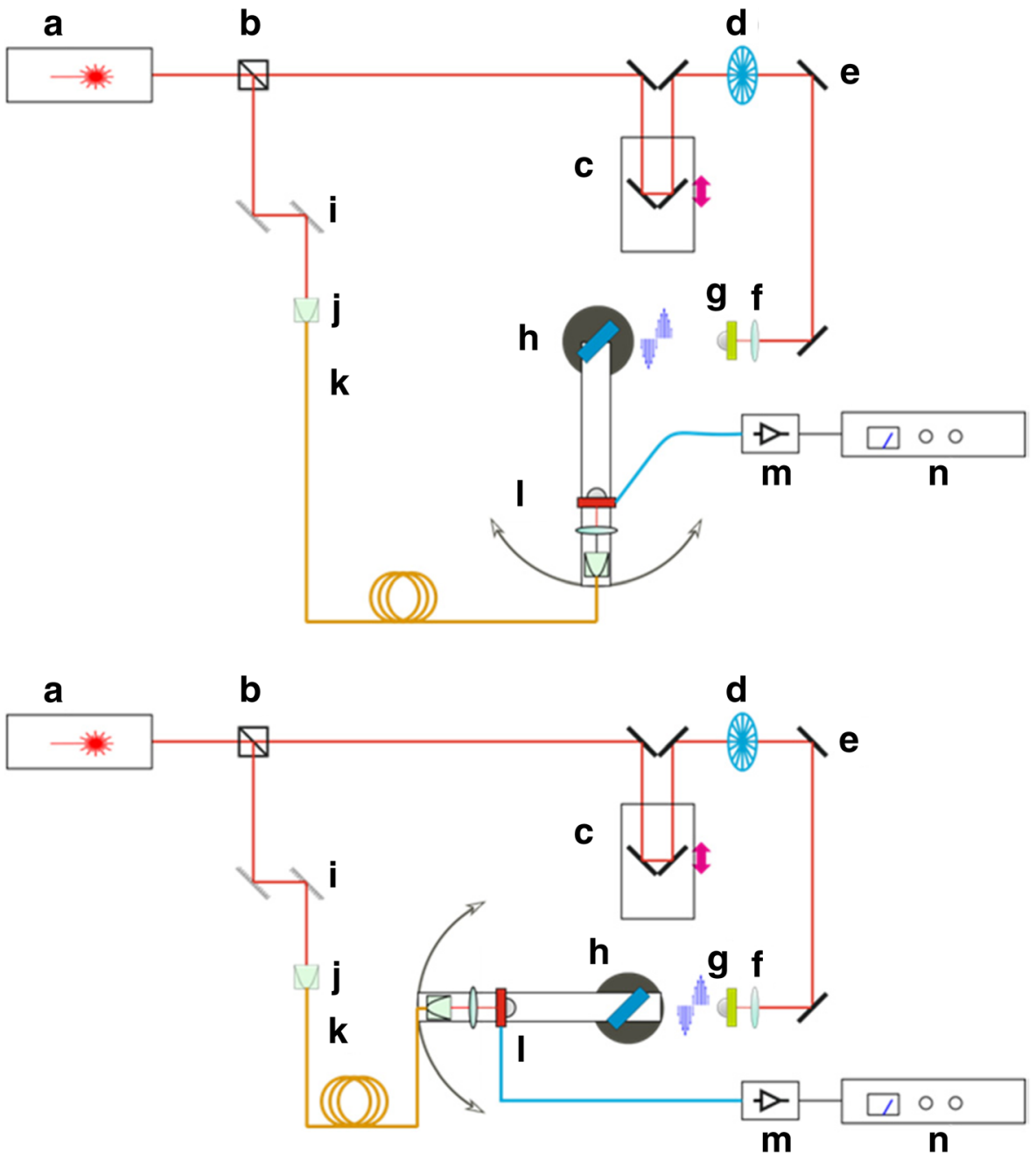

Fig. 4 THz goniometric time-domain spectroscopy setup in reflective (upper) and transmissive mode (bottom). a Femtosecond laser. b Beam splitter. c Delay line. d Chopper. e Mirror. f Lens. $\mathbf{g}$ Emitter. h Sample. i Diffraction grating. j Fiber collimator. k Fiber. I Moving receiver. m Amplifier. n Lock-in 

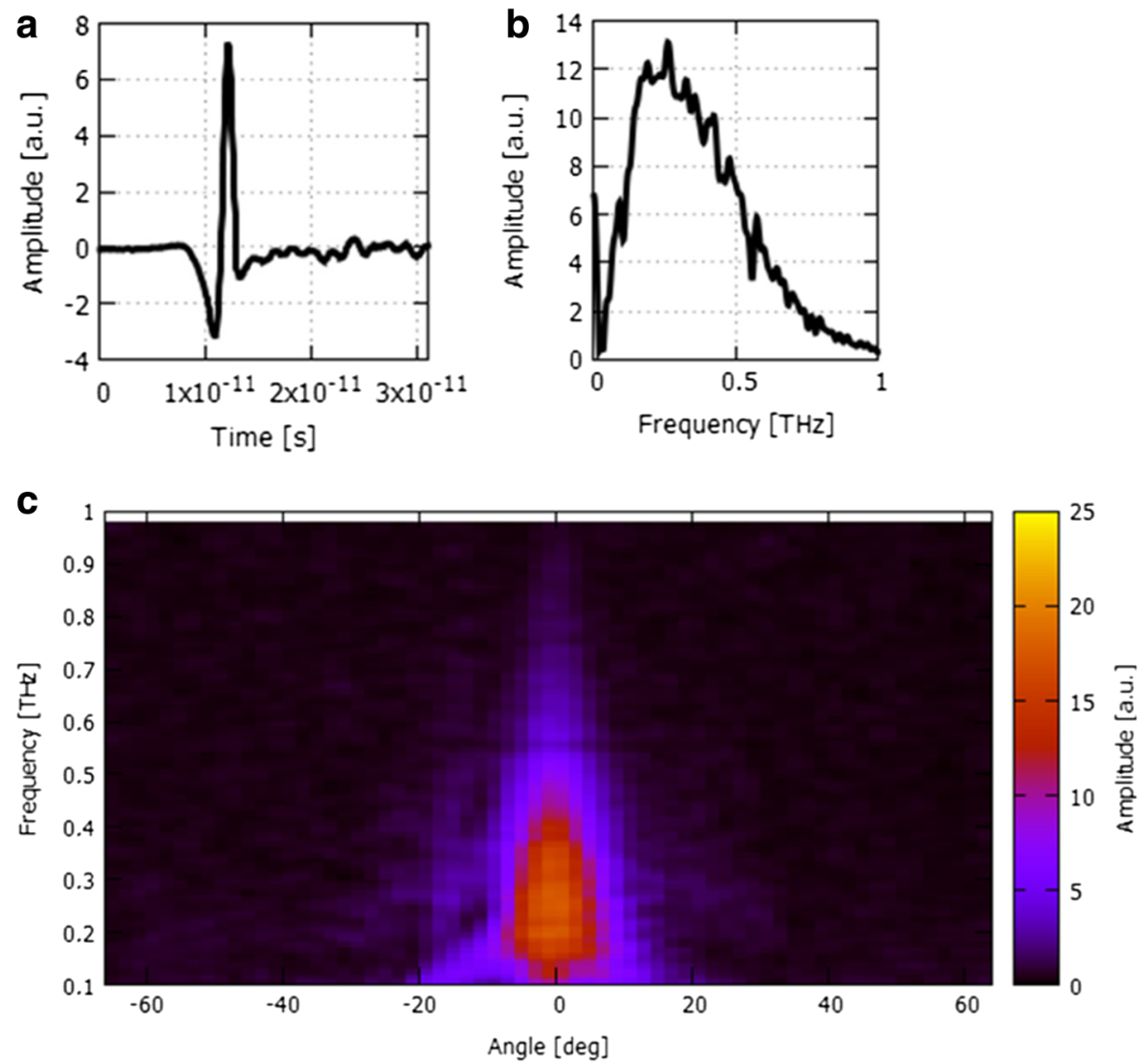

Fig. 5 a Typical temporal THz TDS signal without any sample, its b Fourier transform, and $\mathbf{c}$ angular distribution

wavelength structures for longer wavelengths of the $\mathrm{THz}$ range $[15,16]$. We were able to imprint pyramid-like structures directly on top of the slab in order to form the variable distribution of the effective refractive index.

The distribution of the refractive index has been optimized according to the known methodology of W. H. Southwell [17]. The design was constrained by the precision limit of the 3 -D printer, having the voxel size of $39 \times 39 \times 16 \mu \mathrm{m}$. The AR was designed as a quasi-

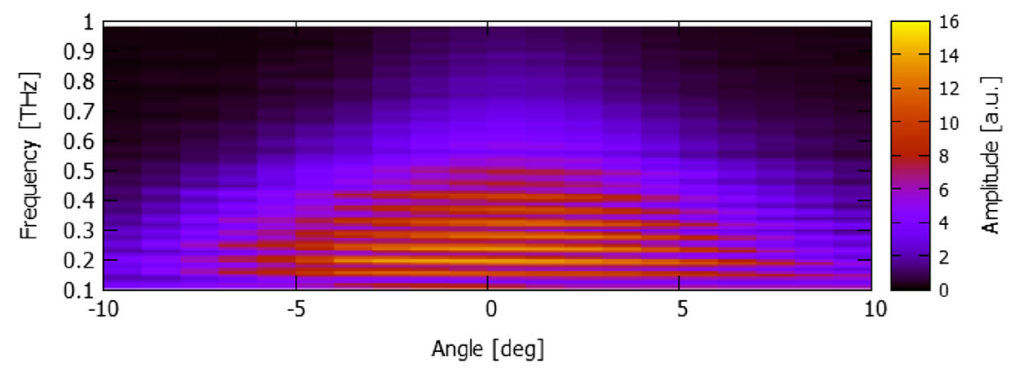

Fig. 6 Angular-frequency representation of the THz signal reflected from the uncoated (reference) slab 


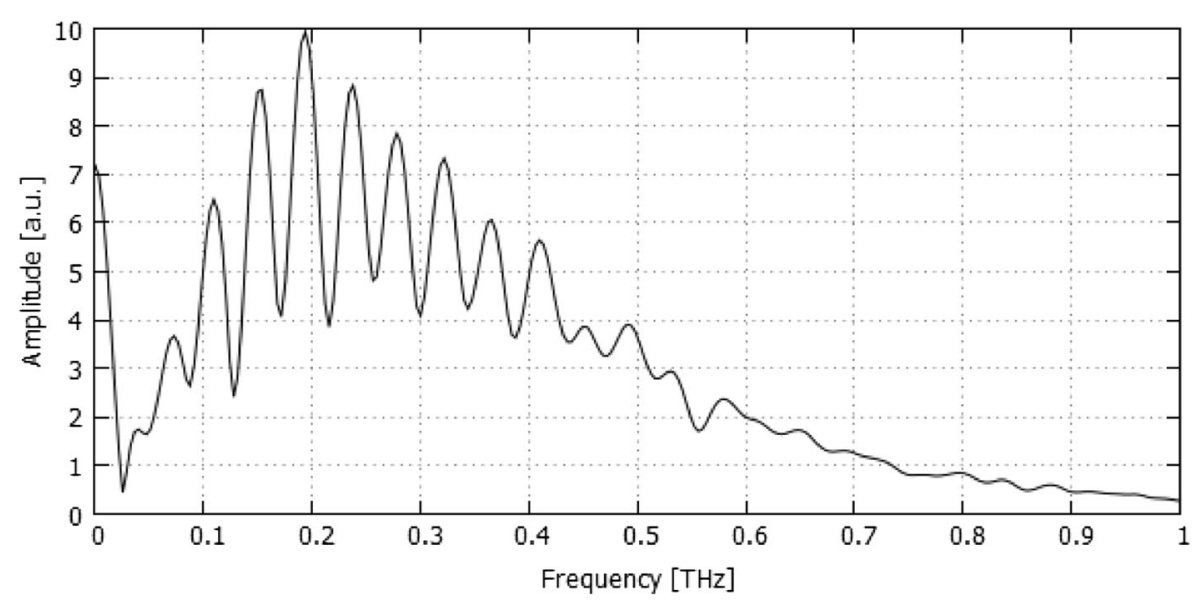

Fig. 7 Fabry-Perot oscillations seen in the averaged signal reflected from the uncoated sample

planar, two-dimensional periodic array of pyramids with the height of $h=2 \mathrm{~mm}$ and spacing equal to $d=1 \mathrm{~mm}$, as shown in Fig. 3 .

The structures were fabricated in the selective laser sintering (SLS) [18] process in PA-12 (polyamide) material, which has the refractive index of 1.64 in the range $0.1-1 \mathrm{THz}$ [19].

\section{Experimental Measurements and Numerical Simulations}

Terahertz time-domain spectroscopy (TDS) systems usually work in in-line mode in which the detector directly observes the emitter, with the sample mounted in between. In our case, the goniometric TDS setup depicted in Fig. 4 was used in order to characterize the spectral transmittance and reflectance of the polyamide slab. The measurements were performed in

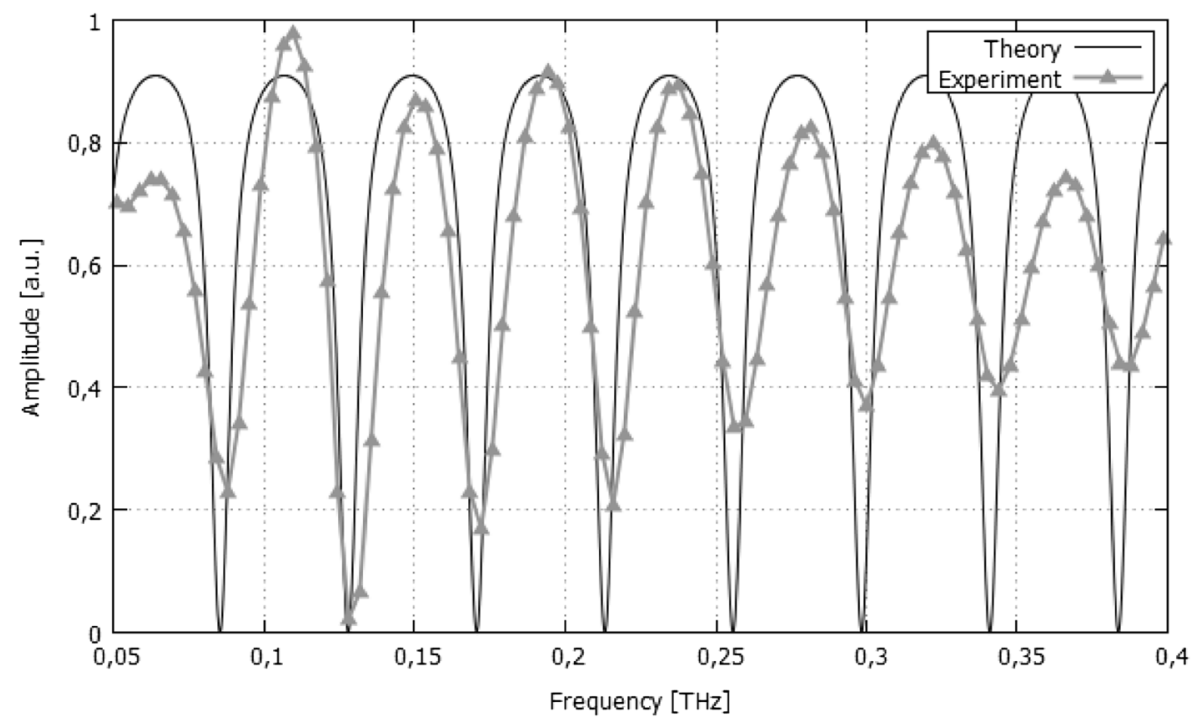

Fig. 8 Fabry-Perot oscillations measured for uncoated PA-12 slab with incidence at $45^{\circ}$ 

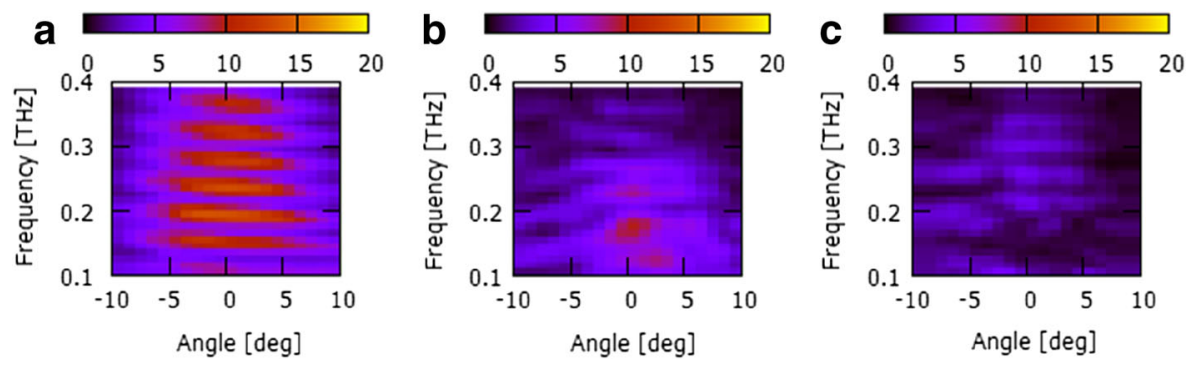

Fig. 9 Experimentally measured amplitude of the THz field reflected from the PA-12 slab. a Without AR structure. b With one-sided AR. c With two-sided AR. The graphs are normalized to the same value

three series: without AR layers, with a one-sided AR layer, and with a two-sided AR layer. The used configuration allowed us to gather the angular distribution of energy around the measured slab in order to detect traces of higher order reflections coming e.g. from the diffraction [20]. It also enabled us to take into account the spectral and angular characteristics of the emitted $\mathrm{THz}$ field and its modification caused by the measured sample.

Low-temperature-grown GaAs semi-conducting antennae illuminated by a femtosecond laser were used as the emitter and the detector. Optical paths of the two interfering beams were precisely adjusted by the mechanical delay line, controlled by a computer. The pumping beam was delivered to the detector via an optical fiber. The induced dispersion is compensated by the pair of diffractive gratings [21]. A correct signal was received in the range $0.1-1 \mathrm{THz}$, which was limited by the poor performance of the source below $0.1 \mathrm{THz}$. The exemplary signal gathered in the time domain, its Fourier transform (FT), and angular distribution are shown in Fig. 5.

The sample was placed in the setup and illuminated by a Gaussian beam with a waist diameter of $30 \mathrm{~mm}$ (as measured for $0.3 \mathrm{THz}$ ). The waist and divergence of the beam obviously varied for different frequencies. The detector was moved along the circle with a diameter of $20 \mathrm{~cm}$ and the sample was located exactly in the center of rotation. The sample was tilted at the angle of $45^{\circ}$ against the beam propagation vector, as seen in Fig. 4. The angular-frequency distribution of the THz signal with the reference flat slab in the sample holder is shown in Fig. 6.

The graph shows the angular shape of the Gaussian beam reflected by the sample, but it also exhibits periodical oscillations proving the presence of the Fabry-Perot effect. In order to

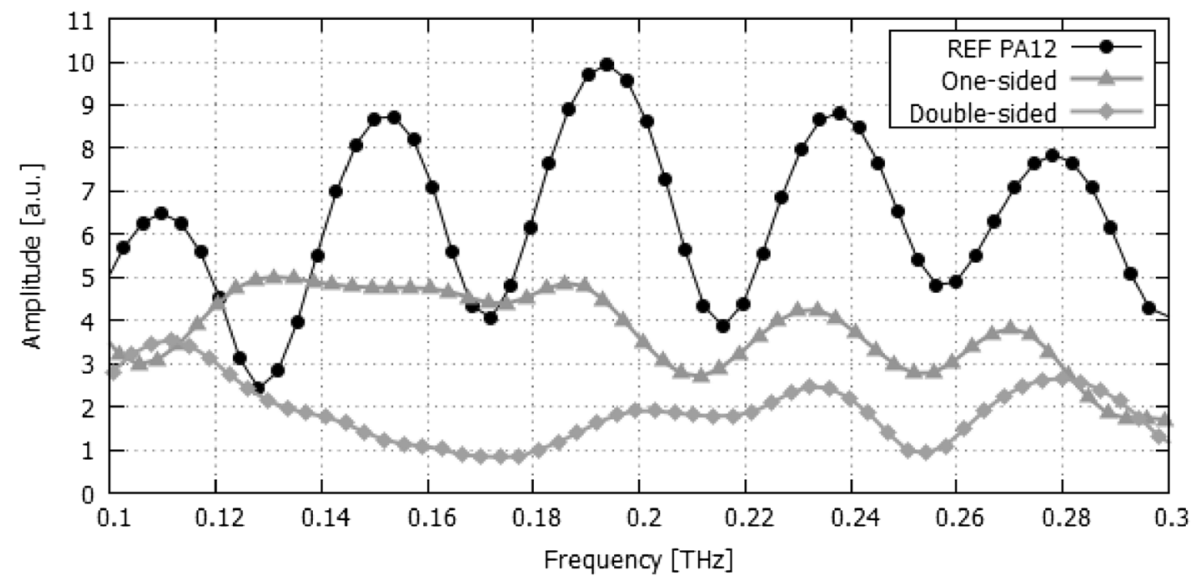

Fig. 10 Averaged amplitude of the reflected $\mathrm{THz}$ beam for different samples 


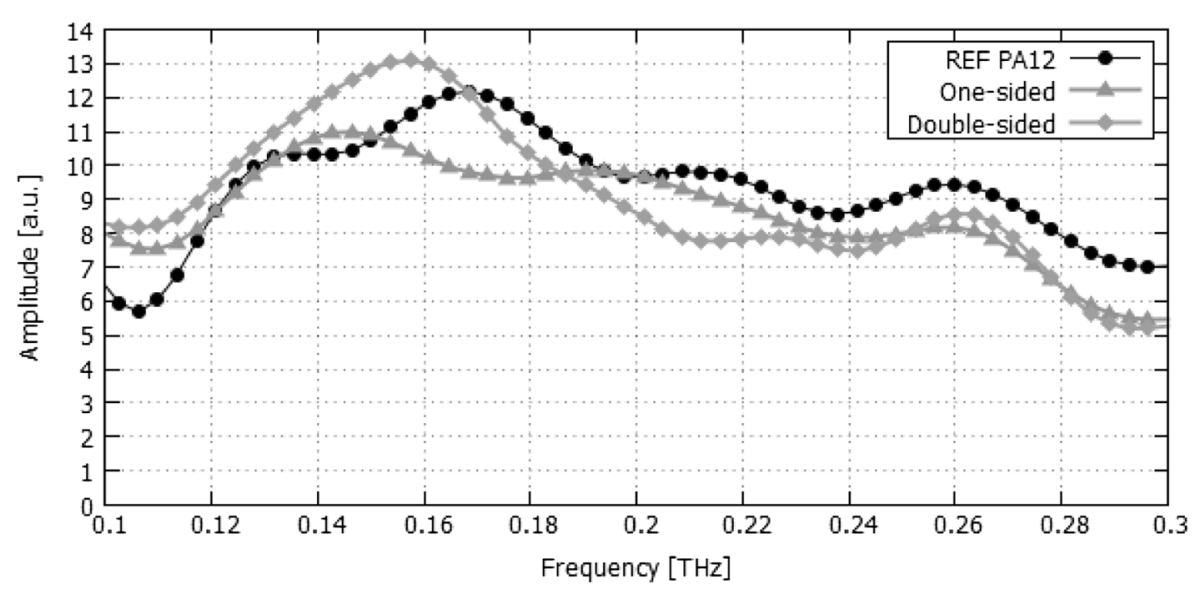

Fig. 11 Amplitude of the $\mathrm{THz}$ beam transmitted in-line through different samples

underline this effect, the signal from all measured angles was averaged for a given frequency and plotted in Fig. 7.

The experimental measurements were then compared with the theoretical curve of F-P oscillations. In the theoretical model, the emitter and detector were assumed to have flat response and the material attenuation was not taken into account. The angle of incidence was $45^{\circ}$ and the sample thickness was the same as in the experiment (i.e. $2 \mathrm{~mm}$ ). Figure 8 shows the expected co-alignment of extremes of both curves, which proved that the observed oscillations are in fact the manifestation of the Fabry-Perot effect.

As the next step, two samples of PA-12 slabs were 3-D printed with the designed AR structure in order to prove the suppression of F-P oscillations for selected spectral regions. They were examined in the same experimental configuration, although this time both reflection and transmission were measured one by one by changing the angle of the detector, as depicted

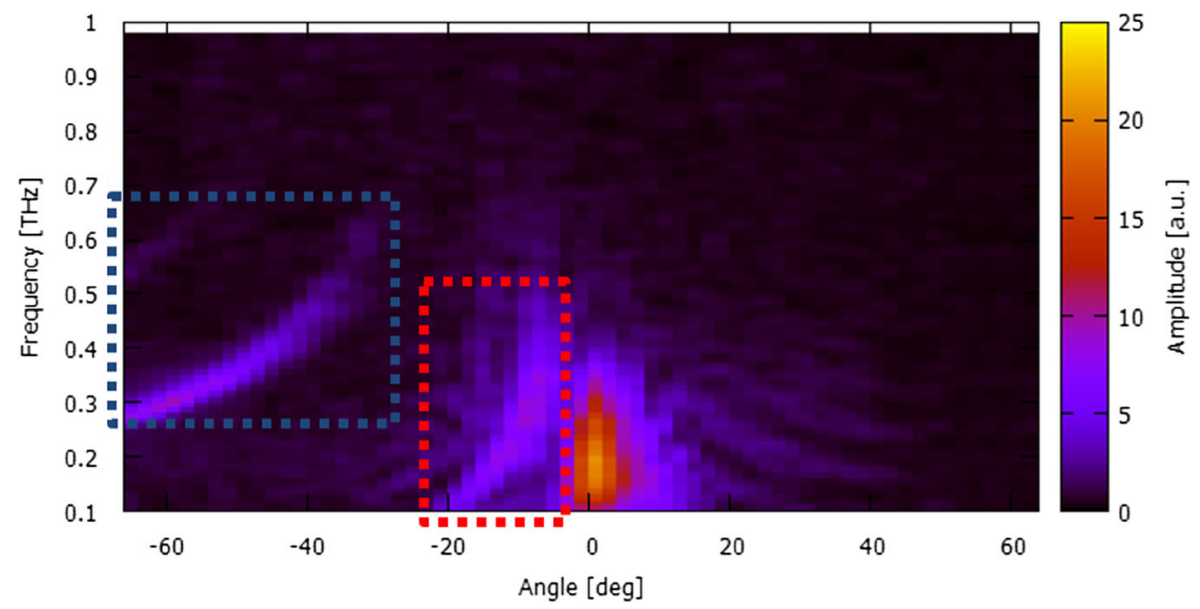

Fig. 12 Amplitude of the transmitted THz field for the wide-angle range, showing diffraction on the left side (for negative angles) in the first diffractive order (marked with a red rectangle) and in the second diffractive order (blue rectangle) 


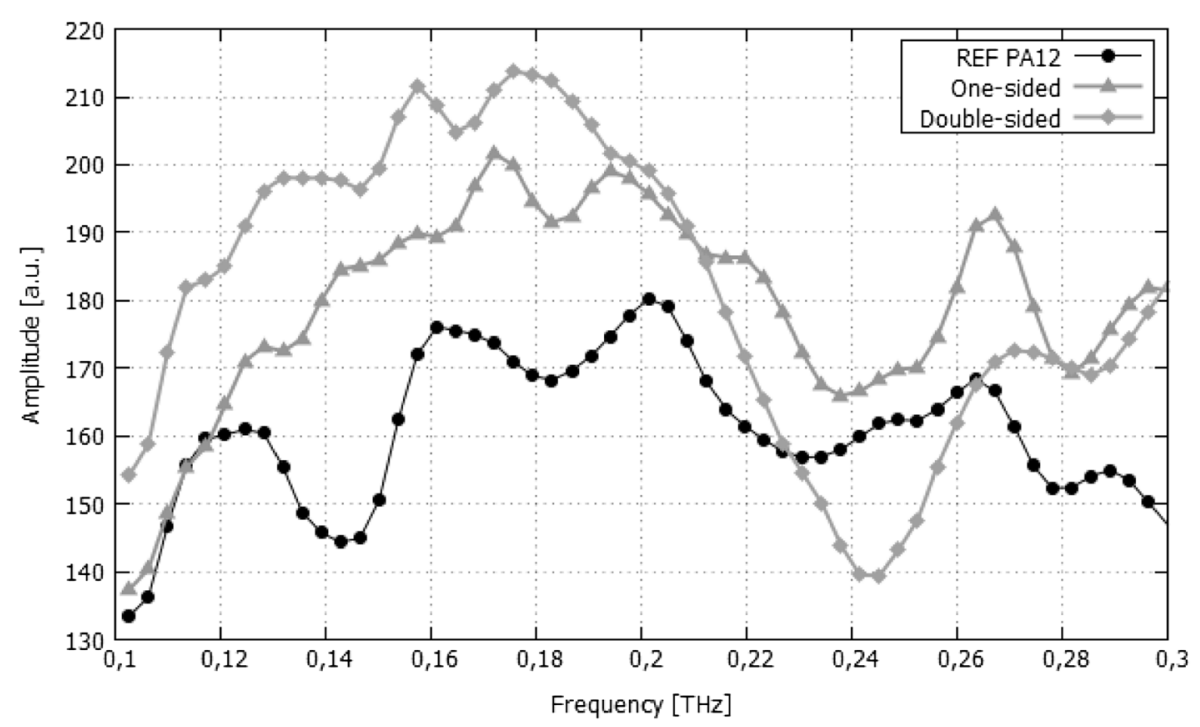

Fig. 13 Amplitude of the transmitted $\mathrm{THz}$ field integrated over a wide angular range

in Fig. 4. The measurements of the reflected field are shown in Fig. 9. Note that the zero angle denotes the direction at which the beam is reflected according to geometrical optics.

The amplitude of the reflected field is significantly reduced due to the use of AR structures. Moreover, the reflected field is more confined near the zero angle. The reduction of reflections in spectral (frequency) domain is presented in Fig. 10, where each value is averaged by angles in the range from $-10^{\circ}$ to $+10^{\circ}$.

Apart from the overall reduction of the amplitude in the reflected beam, the characteristic FP oscillations are highly suppressed, especially for $f<0.2 \mathrm{THz}$. Above $0.2 \mathrm{THz}$, the peak-topeak amplitude of F-P oscillations dropped by a factor of 4 .

The use of 3-D printed AR structures sustained the intensity of the beam transmitted in-line through the PA-12 slab, as expected. Figure 11 shows the amplitude averaged by angles in the range from $-10^{\circ}$ to $+10^{\circ}$, where angle $0^{\circ}$ denotes the direction of propagation of the $\mathrm{THz}$ beam from the emitter.

Below $0.17 \mathrm{THz}$, the transmission is increased, while above $0.17 \mathrm{THz}$, the AR layers have lowered the amount of $\mathrm{THz}$ energy reaching the detector. This is explained by the fact that the used AR structure partly works as a saw-tooth diffractive grating [22-24] for selected (shorter) wavelengths, as it was previously demonstrated e.g. for photo-induced gratings [25]. As a consequence, some of the transmitted energy falls at higher angles due to multi-order

Table 1 Total amplitude of the measured transmitted/reflected THz field shown as percentage of the amplitude from the emitter (numerical results given in parentheses)

\begin{tabular}{clllll}
\hline & $0.1-1 \mathrm{THz}$ & & & $0.12-0.2 \mathrm{THz}$ & \\
\cline { 2 - 3 } \cline { 5 - 6 } & Transmitted field & Reflected field & & Transmitted field & Reflected field \\
\hline REF (no AR layer) & $61.0 \%(60.1 \%)$ & $2.8 \%(2.8 \%)$ & & $73.0 \%(77.1 \%)$ & $2.7 \%(3.3 \%)$ \\
1-sided AR & $65.4 \%(68.0 \%)$ & $1.3 \%(1.2 \%)$ & & $83.6 \%(93.2 \%)$ & $2.0 \%(2.5 \%)$ \\
2-sided AR & $66.7 \%(69.2 \%)$ & $0.8 \%(0.8 \%)$ & & $90.4 \%(98.5 \%)$ & $0.6 \%(0.7 \%)$ \\
\hline
\end{tabular}


Fig. 14 Numerical simulations of the interaction of the upwardpropagating $\mathrm{THz}$ wavefront at 0.1 and $0.3 \mathrm{THz}$ with the test sample without ("REF") and with the onesided and two-sided AR layers. The arrows show the direction of the impinging $\mathrm{THz}$ beam

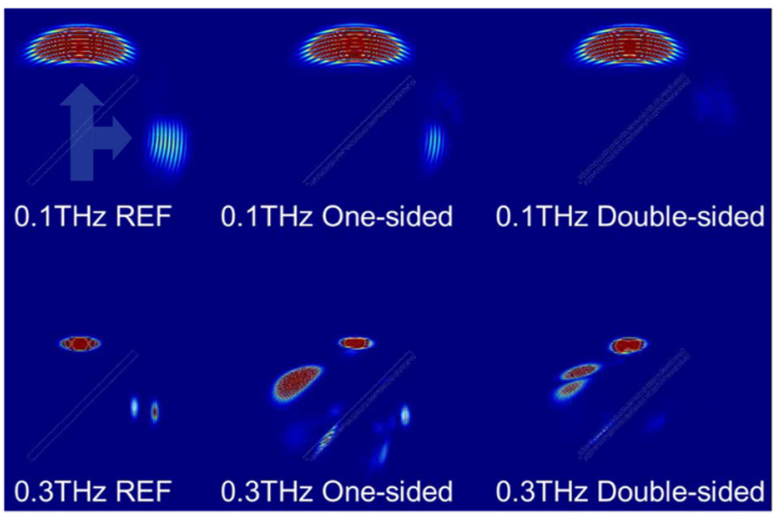

diffraction. In order to confirm this, a wide-angle TDS scan was executed on two-sided AR sample. The results are shown in Fig. 12.

The energy missing in the transmitted characteristics shown in Fig. 11 has been redirected by diffraction into different angles, depending on the relation between the wavelength and the physical periodicity of the printed AR pyramids. For this reason, the proposed AR structures are the most suitable for longer waves (i.e., $0.1-0.2 \mathrm{THz}$ ), which do not feel the periodicity of the $\mathrm{AR}$, but rather interact with the sample as with a sub-wavelength structure having the effective refractive index. 3-D printed AR should perform well for even a lower terahertz range (e.g., $50 \mathrm{GHz}$ ), but those frequencies were out of the operating range of our emitter (see Fig. 5).

When the amplitude of the $\mathrm{THz}$ field transmitted through the sample is integrated over a wider angular range $\left(-60^{\circ}\right.$ to $\left.+60^{\circ}\right)$, the increase of the amplitude is evident and reaches 27 and $36 \%$ at $0.145 \mathrm{THz}$ for one-sided AR and two-sided AR, respectively (see Fig. 13).

Table 1 shows the total amplitude of the transmitted and reflected $\mathrm{THz}$ signals integrated over the entire angular range. In the optimal range of frequency between 0.12 and $0.2 \mathrm{THz}$, the use of 3-D printed AR structure increased the transmitted amplitude by $14 \%$ (one-sided AR)

Fig. 15 Multiple passes of the radiation inside the sample, contributing to the increased absorbance

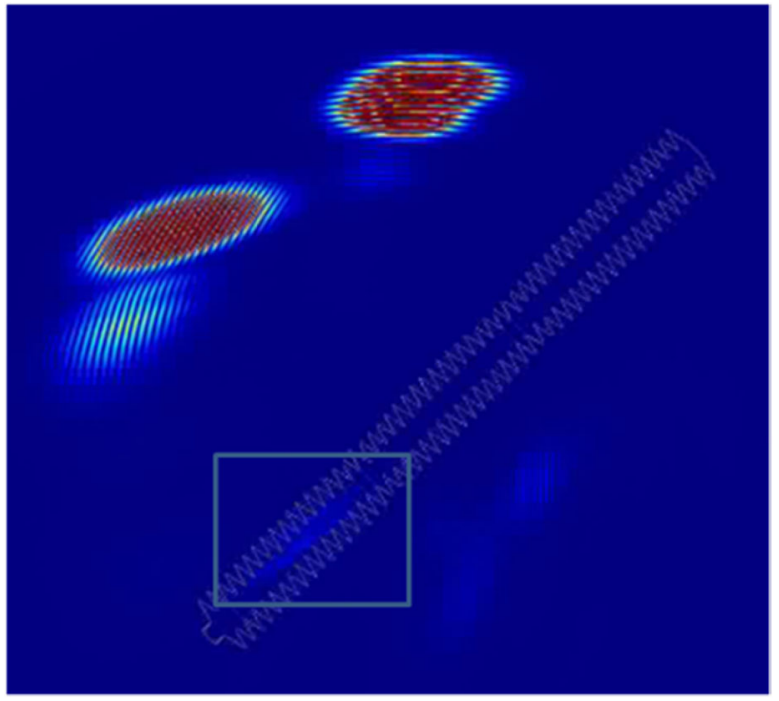


and $23 \%$ (two-sided AR). The reflected amplitude was decreased by $26 \%$ (one-sided AR) and $77 \%$ (two-sided AR), which is a significant improvement taking into account the ease of design and fabrication.

The presented quantitative and qualitative results are in a good agreement with numerical simulations performed using finite-difference time-domain (FDTD) method. Figure 14 compares the amplitude of transmitted, diffracted, and reflected $\mathrm{THz}$ fields for $0.1 \mathrm{THz}$ (where the AR structure should work as an effective medium layer) and for $0.3 \mathrm{THz}$ (where the diffraction is strongly visible). The integrated values from the simulations are also given in Table 1.

According to numerical results in Fig. 14, the use of AR layer has allowed the redirection of $\mathrm{THz}$ energy from the reflected beam to the transmitted/diffracted beam, leading to sustained transmission and strongly attenuated unwanted reflections, especially when the two-sided AR structure was used. These conclusions fully agree with the previously shown experimental outcome. Moreover, the matching values of experimental and numerical integrals in Table 1 prove that the assumed material absorption coefficient of $\alpha=1 \mathrm{~cm}^{-1}$ [26] was correct. Based on the above results, the effective thickness of the uncoated sample was calculated to be $6.3 \mathrm{~mm}$. It was longer than its physical width as the result of multiple reflections inside the PA12 slab, which was also observed in simulations (see Fig. 15). The application of the two-sided AR layer has decreased the effective thickness of the sample from 6.3 to $2.0 \mathrm{~mm}$.

\section{Discussion and Conclusions}

Additive manufacturing proves to be a promising technology for structuring of optical elements for the wavelengths in the millimeter range due to its ease of design and prototyping [26-28]. Especially for longer wavelengths around $0.1 \mathrm{THz}$, there is an attractive possibility of direct printing of sub-wavelength kinoform-like structures. Anti-reflection structures fabricated using this technology were tested in THz-TDS setup with goniometric angular measurements of both transmitted and reflected fields. AR layers successfully reduced the reflected energy and suppressed internal reflections leading to spurious Fabry-Perot interference. Moreover, they improved the total transmittance of the sample, but mostly for longer waves in the range where the diffraction of the THz beam on the AR structure was negligible. The experimental results were in good agreement with numerical simulations. The performance of the proposed AR layers may be improved in future work by optimizing the groove profile and periodicity of the pyramid design. Nevertheless, in the THz experiments, the most disturbing effect is the presence of unwanted multiple reflections, which can be strongly suppressed by the proposed solution.

Acknowledgements The authors would like to thank Dr Paweł Jung for his valuable assistance.

Open Access This article is distributed under the terms of the Creative Commons Attribution 4.0 International License (http://creativecommons.org/licenses/by/4.0/), which permits unrestricted use, distribution, and reproduction in any medium, provided you give appropriate credit to the original author(s) and the source, provide a link to the Creative Commons license, and indicate if changes were made.

\section{References}

1. H. K. Raut, V. A. Ganesh, A. S. Nairb, and S. Ramakrishna, Energy Environ. Sci., 4, 3779 (2011). 
2. G.Savinia, P.C.Hargrave, Infrared Millimeter and Terahertz Waves (IRMMW-THz), 2010 35th International Conference on. IEEE, 2010.

3. C. Brückner, B. Pradarutti, O. Stenzel, R. Steinkopf, S. Riehemann, G. Notni, A. Tünnermann, Opt. Express 15,779 (2007).

4. P. F. Goldsmith, Quasioptical Systems (IEEE Press, New York, 1998).

5. C. R. Englert, B. Manfred, and M. Hansjörg, IEEE transactions on geoscience and remote sensing 37.4, 1997-2003 (1999).

6. C. Brückner, B. Pradarutti, S. Riehemann, O. Stenzel, R. Steinkopf, A. Gebhardt, G. Notni, A. Tünnermann, Proc. SPIE 6194, 61940N1 (2006).

7. X. Liu, K. Fan, I. V. Shadrivov, W. J. Padilla, Opt. Express 25, 191 (2017).

8. A. J. Gatesman, J. Waldman, M. Ji, C. Musante, and S. Yngvesson, IEEE Microw. Guid. Wave Lett., 10, (2000).

9. E. Gerecht, et al., Microwave Symposium Digest. 2000 I.E. MTT-S International. Vol. 2. IEEE (2000).

10. K. Neeraj et al., Opt. Lett. 42, $1764-1767$ (2017).

11. F. S. Crawford, Berkeley physics course v. 3: Waves (New York, NY : McGraw-Hill, 1968).

12. S. G. Lipson, H. Lipson, D. S. Tannhauser, Optical Physics 3rd ed. (London: Cambridge U. P., 1995).

13. A. D. Squires, E. Constable, and R. A. Lewis, J Infrared Milli Terahz Waves 36.1, $72-80$ (2015).

14. J. Suszek et al., Opt. Expr. 22, 3137-3144 (2014).

15. J. Suszek, et al., IEEE transactions on Terahertz Science and Technology 5, 314-316 (2015).

16. S. F. Busch, et al. J Infrared Milli Terahz Waves 35.12 993-997 (2014).

17. W. H. Southwell, JOSA A 8, 549 (1991).

18. C. Deckard, "Method and apparatus for producing parts by selective sintering", U.S. Patent 4,863,538.

19. X. Yan, P. Gu, Comput. Aided Des. 28, 307 (1996).

20. Y. Monnai, et al., Opt. Express 21, 2347-2354 (2013).

21. S. Rulliere, Femtosecond Laser Pulses Principles and Experiments (Springer Science, Berlin, 2005).

22. J. Bomba, A. Sobczyk, A. Siemion, K. Świtkowski, C. Jastrzębski, A. Siemion, J. Suszek, M. Sypek, Photon. Lett. Pol. 4, 121 (2012).

23. B. Scherger, N. Born, C. Jansen, S. Schumann, M. Koch, and K. Wiesauer, IEEE Trans. on THz Science and Techn. 2, 556 (2012).

24. J. L. Coutaz, et al., Acta Phys. Pol. A 107, 26-37 (2005).

25. I. Chatzakis, et al., App. Phys. Lett. 103, 043101 (2013).

26. K. Szkudlarek, M. Sypek, G. Cywiński, J. Suszek, P. Zagrajek, A. Feduniewicz-Żmuda, D. B. But, Opt. Express, 24, 20119 (2016).

27. R. Vilar, R. Czarny, M.S.L. Lee, B. Loiseaux, M. Sypek, M. Makowski, C. Martel, T. Crepin, F. Boust, R. Joseph, K. Herbertz, T. Bertuch, J. Marti, IEEE Microwave Magazine 15, 121 (2014).

28. K. Liebert et al. J Infrared Milli Terahz Waves 38, 1-12 (2017). 